(C) 1984. The Genetical Society of Great Britain

\title{
SELECTIVE EFFECTS OF THE GENETIC BACKGROUND AND ETHANOL ON THE ALCOHOL DEHYDROGENASE POLYMORPHISM IN DROSOPHILA MELANOGASTER
}

\author{
J. G. OAKESHOTT, ' J. B. GIBSON' AND S. R. WILSON ${ }^{2}$ \\ 1 Department of Population Biology, Research School of Biological Sciences, \\ Australian National University, GPO Box 475, Canberra, ACT 2601, Australia \\ ${ }^{2}$ Department of Statistics, Research School of Social Sciences, \\ Australian National University, GPO Box 4, Canberra, ACT 2601, Australia
}

Received 5.xii.83

\section{SUMMARY}

\begin{abstract}
Eight freshly caught Australasian mass collections ranging in Adh $F$ frequency from 4 to 96 per cent were each divided into eight selection lines. Two selection lines from each base population were put on one of four types of mediumstandard food supplemented with 0 per cent, 3 per cent, 6 per cent or 9 per cent ethanol. After 30 generations the tolerance of 6 per cent and 9 per cent selection lines on a test dose of 9 per cent ethanol was greater than that of the 0 per cent lines on this dose. The tolerance of the 3 per cent lines on this dose was less than that of the 0 per cent lines. There were no significant differences in the tolerance responses across the eight base populations but in only one did Adh frequencies diverge among the four ethanol selection environments: $F$ frequencies in the Brisbane 9 per cent lines were higher than in the Brisbane 0 per cent, 3 per cent and 6 per cent lines. Although the selection affecting Adh did not generally differ among the four selection environments, it differed highly significantly among the eight base populations. The equilibrium $F$ frequencies predicted from the maximum likelihood estimates of the selection coefficients were in close agreement with the frequencies observed in the original collections. The only aspect of the coefficients which was consistent across base populations was FS heterozygote superiority.
\end{abstract}

\section{INTRODUCTION}

For over a decade the polymorphism for the electrophoretically detectable alcohol dehydrogenase ( $A d h) F$ and $S$ alleles of Drosophila melanogaster has been one of the most promising systems for studying the selective forces operating on enzyme polymorphisms. There is good evidence that the two alleles are not neutral to selection in wild populations; large-scale and complementary latitudinal clines have been reported on all continents investigated (Johnson and Schaffer, 1973; Malpica and Vassallo, 1980; Oakeshott et al., 1982a; David, 1982). The mechanism of selection has seemed amenable to study because of the presumed role of alcohol dehydrogenase in the tolerance to, and utilisation of, ingested ethanol.

The evidence from natural populations is however ambiguous concerning the selective effects of ethanol on the Adh locus. Microgeographic variation in allele frequencies in, and around, wineries (Briscoe et al., 1975; Hickey and McLean, 1980; Marks et al., 1980; McKenzie and McKenie, 1981 ) is not generally related to differences in the ethanol concentrations of the substrates (Gibson et al., 1981). In terms of macrogeographic variation, David and Bocquet (1975) and Parsons and Stanley (1981) have some evidence that $\mathrm{ADH}$ activity and ethanol tolerance are lower in populations 
close to the equator than in cold temperate regions; this parallels the clinal distribution in $F$ frequency. On the other hand, an analysis of isofemale lines drawn from diverse latitudes by Anderson (1982) suggested that there is little if any inherited causal relationship between latitudinal variation in $F$ frequency and ethanol tolerance.

Laboratory experiments directed at the $A d h /$ ethanol fitness relationships have lent some support to the contention that ingested ethanol does select on Adh. Several multi-generation experiments have suggested that $F$, which produces at least twice the ADH activity of $S$, reaches higher equilibrium frequencies in populations kept on ethanol impregnated food than in those kept on food without added ethanol (van Delden, 1982 for a review). But once again the total experimental results are ambiguous; Gibson and Oakeshott (1982) have pointed out that higher $F$ frequencies have generally only been obtained with populations which have spent at least a year in the laboratory prior to the experiment (Gibson, 1970; Bijlsma-Meeles and van Delden, 1974; van Delden et al., 1978; Oakeshott, 1979; Cavener and Clegg, 1981 ; Vigue et al., 1982).

In our previous experiments with freshly caught mass collections of winery populations from the Hunter Valley (NSW, Australia), no consistent differences in $F$ frequency on media with and without added ethanol were found (Gibson et al., 1979; Oakeshott et al., 1983). The experiment described in the present paper tests the generality of this finding by investigating the responses to ethanol made by populations collected from various habitats over a wide range of latitudes. The analytical methods of Wilson et al., (1982) are used to calculate the selection coefficients operating on Adh in the presence of ethanol concentrations ranging from 0 per cent, through 3 per cent (which longevity data suggest is a metabolic benefit; Parsons and Stanley, 1981) to 6 per cent and 9 per cent (which mortality data indicate is a metabolic cost; Oakeshott et al., 1980).

\section{MATERIALS AND METHODS}

The experiment was initiated with flies from isofemale lines set up from recently collected females at eight Australasian localities covering a wide range of latitudes and habitat types (table 1). Flies from twenty isofemale lines from each locality were combined after between two and five generations in the laboratory to produce eight base populations for the experiment. Population sizes were expanded during the next two generations and then eight random samples of 40 adult males and 40 adult females were taken from each base population to establish eight selection lines. Two of these eight selection lines were transferred to each of four different types of medium: standard medium supplemented with $0,3,6$ or 9 per cent $(v / v)$ ethanol (recipe in Oakeshott and Gibson, 1981).

The total of 64 selection lines were thereafter maintained on their respective media types for 30 discrete four-week generations at $22 \pm 2^{\circ} \mathrm{C}$. All cultures were made in $250 \mathrm{ml}$ bottles each containing $50 \mathrm{ml}$ medium. For each selection line forty males and forty females were transferred to a new bottle each generation; in exceptional cases where fewer flies were produced all flies produced were used. The means and standard errors of the numbers of flies transferred per generation, averaged over all selection lines and generations, were $79 \cdot 5 \pm 0 \cdot 1$ for 0 per cent, $79 \cdot 5 \pm 0 \cdot 1$ for 3 per 


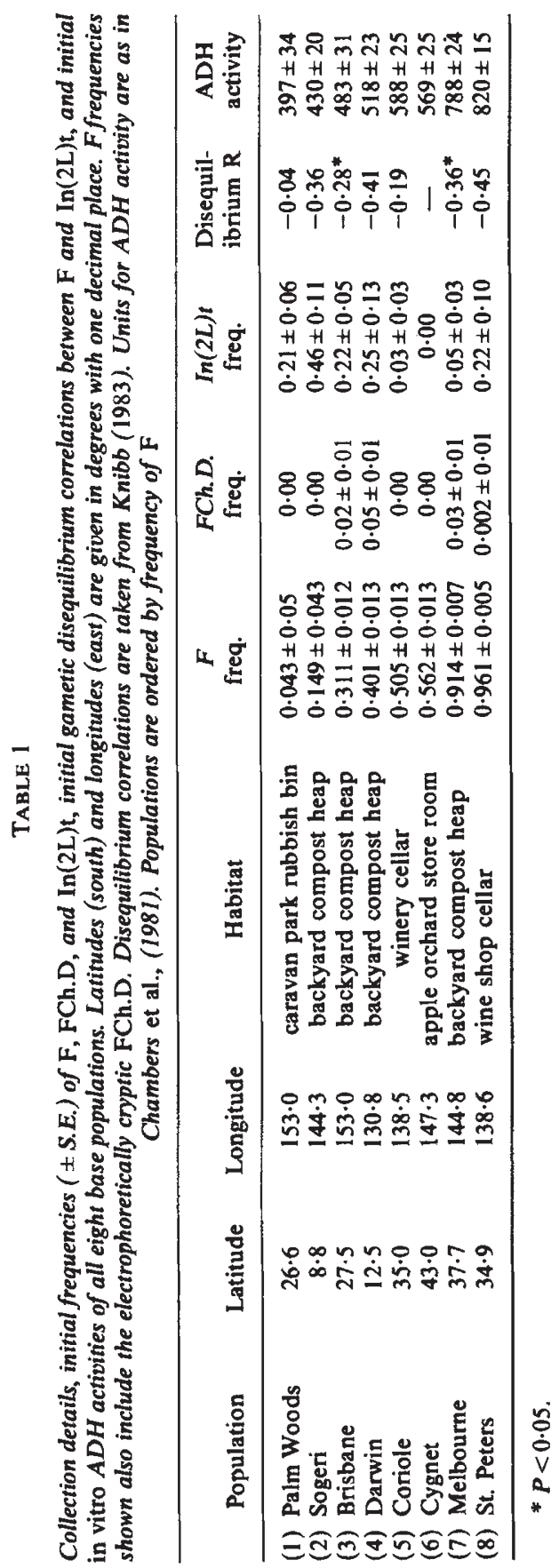


cent, $79 \cdot 0 \pm 0 \cdot 1$ for 6 per cent and $77 \cdot 1 \pm 0 \cdot 2$ for the 9 per cent ethanol media. These numbers did not differ significantly among the eight base populations. Three days after transfer the surviving parents in each generation were counted and removed.

$F$ and $S$ frequencies were determined in all selection lines at generations $0,10,20$ and 30. The electrophoretic methods of Lewis and Gibson (1978) were used and the means and standard errors of the numbers of $A d h$ genes scored in each experimental line were $151 \pm 1$ at generation $0,283 \pm 5$ at generation 10,258 \pm 8 at generation 20 and $182 \pm 2$ at generation 30 . These numbers were consistent across the four selection environments and, for generations 0,10 and 20, always included the parents used for the next generation. The generation 0 sample was also scored for the electrophoretically cryptic thermostable variant FCh.D., using the methods of Wilks et al. (1980).

ADH activities were determined in samples taken at generation 0 from the expanded base populations from which the selection lines were established. Crude extracts of 7-day old adult males were assayed using the spectrophotometric methods of Chambers et al. (1981) with propan-2-ol as substrate.

In order to assess the response to selection in terms of ethanol tolerance, egg-to-adult and adult survival on 9 per cent ethanol food were measured on subcultures of all generation 30 selection lines. The subcultures were produced by transferring a sample of 50 generation 30 adults from each selection line to standard medium without added ethanol. Their progeny were collected as adults over a three day period and aged for a further three days on standard medium lacking ethanol. To measure adult survival, single sex cohorts of 20 were then put on standard medium +9 per cent ethanol and after three more days the survivors were counted. To measure egg-toadult survival other cohorts of females were left for 12 hours to lay eggs on standard medium +0 per cent ethanol. The eggs were then transferred in groups of 20 to standard medium +9 per cent ethanol. The flies developing from these eggs were counted when they emerged as adults.

The conventions for nomenclature and the methods for the statistical analysis were as described in Wilson et al. (1982), and Wilson and Oakeshott (1984). Each selection line was denoted by a three-symbol code $(b, e, r)$ uniquely defining it in terms of three factors: the base population from which it was derived $(b=1,2, \ldots, 7,8)$, the ethanol environment to which it was allocated $(e=0,3,6,9)$ and the replicate number within its particular $b-e$ combination $(r=1,2)$. Selection coefficients were estimated by maximum likelihood methods for each selection line and for various groups of them defined by $(b, e, r)$ values. Differences between the coefficients for the different selection lines and their groups were assessed by analysis of deviance.

\section{REsults}

Because the eight base populations were drawn from a wide range of latitudes, their initial $F$ and $S$ frequencies differed considerably, as did the frequency of the linked chromosome inversion $\operatorname{In}(2 L) t$ (table 1$)$. The tendency for the initial frequencies of $S$ and $\operatorname{In}(2 L) t$ to decline with increasing latitude was consistent with their previously documented latitudinal clines 
(Knibb et al., 1981; Oakeshott et al., 1982a). As reported by Knibb (1983), $F$ and $S$ were consistently in gametic disequilibrium with $\operatorname{In}(2 L) t$ : chromosomes carrying $\operatorname{In}(2 L) t$ generally also bore $S$. The initial frequencies of the electrophoretically cryptic $F C h . D$. allele varied between 0.00 and 0.05 across the eight base populations and, as reported in Wilks et al. (1980), were not clinally related to latitude. ADH activities were highly correlated with $F$ frequencies $(r=0.86, P<0.001)$ and, after correction for the covariate effects of $F$ frequencies, did not differ significantly between the eight base populations $\left(F_{(7.80)}=0.24, P>0.05\right)$.

The percentage survival of parents in selection lines on 0 per cent and 3 per cent ethanol consistently exceeded 95 per cent throughout the 30 generations of the experiment. The same was true of the 6 per cent selection lines in the first 20 generations. However the percentage survival of parents in selection lines on 9 per cent ethanol increased regularly with generation number, except for a sudden but temporary drop between generations 17 and 21 (fig. 1). A similar, temporary drop occurred at this time for the 6

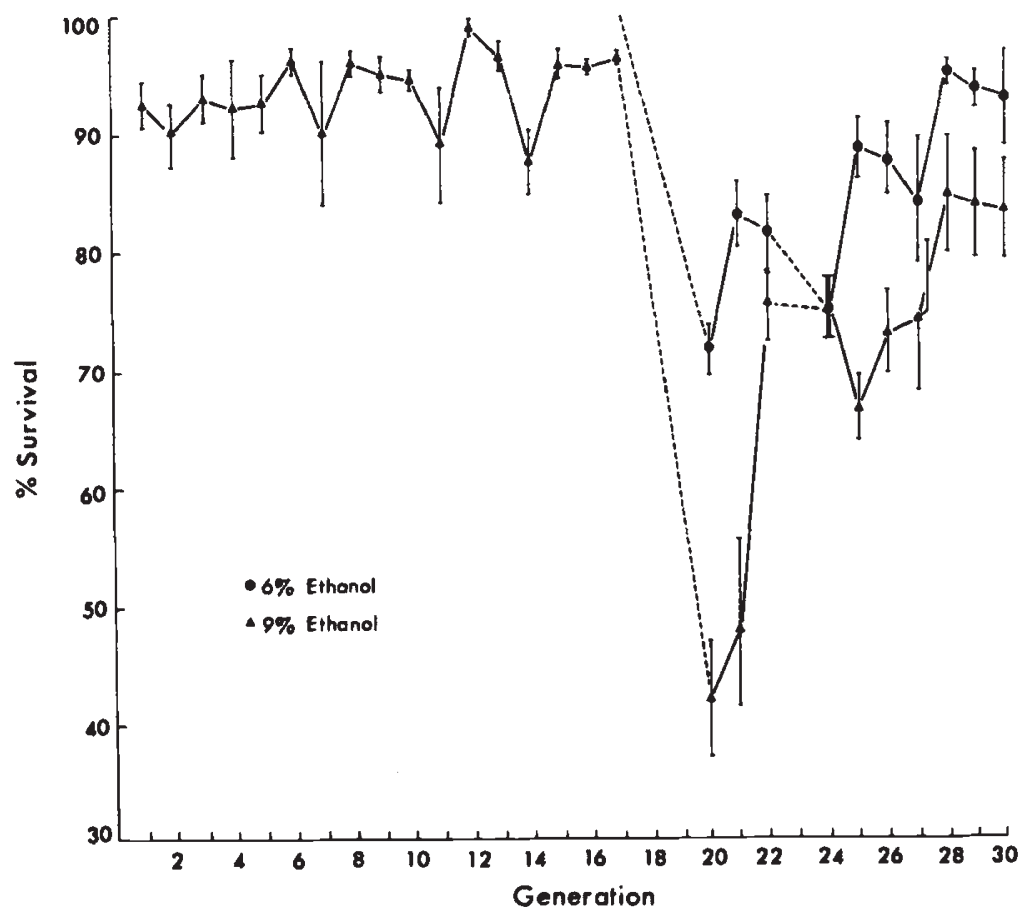

FIG. 1. Survival percentages of the parents used over the 30 generations in the 9 per cent ethanol selection lines, and over the last 10 generations in the 6 per cent ethanol selection lines.

per cent selection lines. The drop corresponded to a decrease in the temperature to which the media was cooled $\left(\sim 48^{\circ} \mathrm{C}\right.$ after versus $\sim 55^{\circ} \mathrm{C}$ previously) before the ethanol was added. It was suspected that less of the ethanol evaporated immediately after its addition in the later generations.

At generation 30 the egg-to-adult and adult male and female survival percentages of all selection lines were measured after test doses of 9 per 


\section{TABLE 2}

Means ( \pm S.E.) over all base populations and replicates of egg-to-adult and adult male and female percentage survivals on test doses of 9 per cent ethanol media. The flies tested are generation 30 subcultures of all 0 per cent, 3 per cent, 6 per cent and 9 per cent selection lines. The significance of differences from the values for the 0 per cent lines are indicated for the 3 per cent, 6 per cent and 9 per cent lines ( $n s=$ not significant, $* \mathrm{P}<0.05),{ }^{* *} \mathrm{P}<0.01$ )

\begin{tabular}{cccc}
\hline & \multicolumn{3}{c}{ Percentage survival on the 9 per cent ethanol test dose } \\
\cline { 2 - 4 } $\begin{array}{c}\text { Selection } \\
\text { environment }\end{array}$ & Egg-to-adult & Adult male & Adult female \\
\hline$e=0 \%$ & $51 \pm 7$ & $93 \pm 1$ & $85 \pm 2$ \\
$e=3 \%$ & $34 \pm 6^{*}$ & $89 \pm 2^{* *}$ & $86 \pm 2^{\text {ns }}$ \\
$e=6 \%$ & $59 \pm 8^{\text {ns }}$ & $95 \pm 1^{\text {ns }}$ & $89 \pm 2^{*}$ \\
$e=9 \%$ & $69 \pm 6^{*}$ & $95 \pm 1^{\text {ns }}$ & $90 \pm 2^{*}$ \\
\hline
\end{tabular}

cent ethanol food (table 2). The selection responses indicated by all three measures of tolerance were homogeneous across the eight base populations $\left(F_{(3,40)}=0.30, P>0.05\right.$ for egg-to-adult, $F_{(3,230)}=0.79, P>0.05$ for adult male and $F_{(3,229)}=0.29, P>0.05$ for adult female survival). The 9 per cent selection lines had higher survival percentages than the 0 per cent lines for all three measures of tolerance. The 6 per cent lines also had higher survival percentages than the 0 per cent lines for all three measures, but only for adult females was the difference significant. The 3 per cent lines were not significantly different from the 0 per cent lines in the survival percentages of adult females and were significantly lower than the 0 per cent lines in the survival of preadults and adult males.

The trajectories of $A d h$ genotype and allele frequencies over the 30 generations are shown for all 64 selection lines in the De Finetti diagrams in fig. 2. Preliminary analysis of the genotype frequencies was suggestive of heterozygote advantage. In a highly significant majority, 155, of the 213 segregating samples scored, there was an excess of heterozygotes above Hardy-Weinberg expectations. In 23 cases this excess was statistically significant. There were only five cases of significant heterozygote deficiency. The trend for heterozygote excess was consistent across the four ethanol environments and eight base populations (except for St Peters $(b=8)$, for which an $F$ frequency of 100 per cent was reached in all 8 selection lines by generation 10 ).

Preliminary analysis of the allele frequencies revealed that $F$ frequency was higher at generation 30 than generation 0 in a significant majority, 46, of the 64 selection lines. This trend was generally consistent across the eight populations, although it was most obvious in St Peters. It was also generally maintained across the four ethanol environments; of the 16 selection lines in each environment, 12 showed a rise in 0 per cent ethanol lines, 8 in 3 per cent lines, 13 in 6 per cent lines and 13 in 9 per cent lines. The weakness of the trend was however indicated by a turning point analysis. In just over half, 56 of 96 possible cases, the direction of $F$ frequency change was reversed in successive 10 -generation intervals.

Consistent with these reversals, fig. 2 also shows a high degree of variation between replicate selection lines in both the magnitude and direction of the trajectories. Notwithstanding the significant majority of heterozygote 

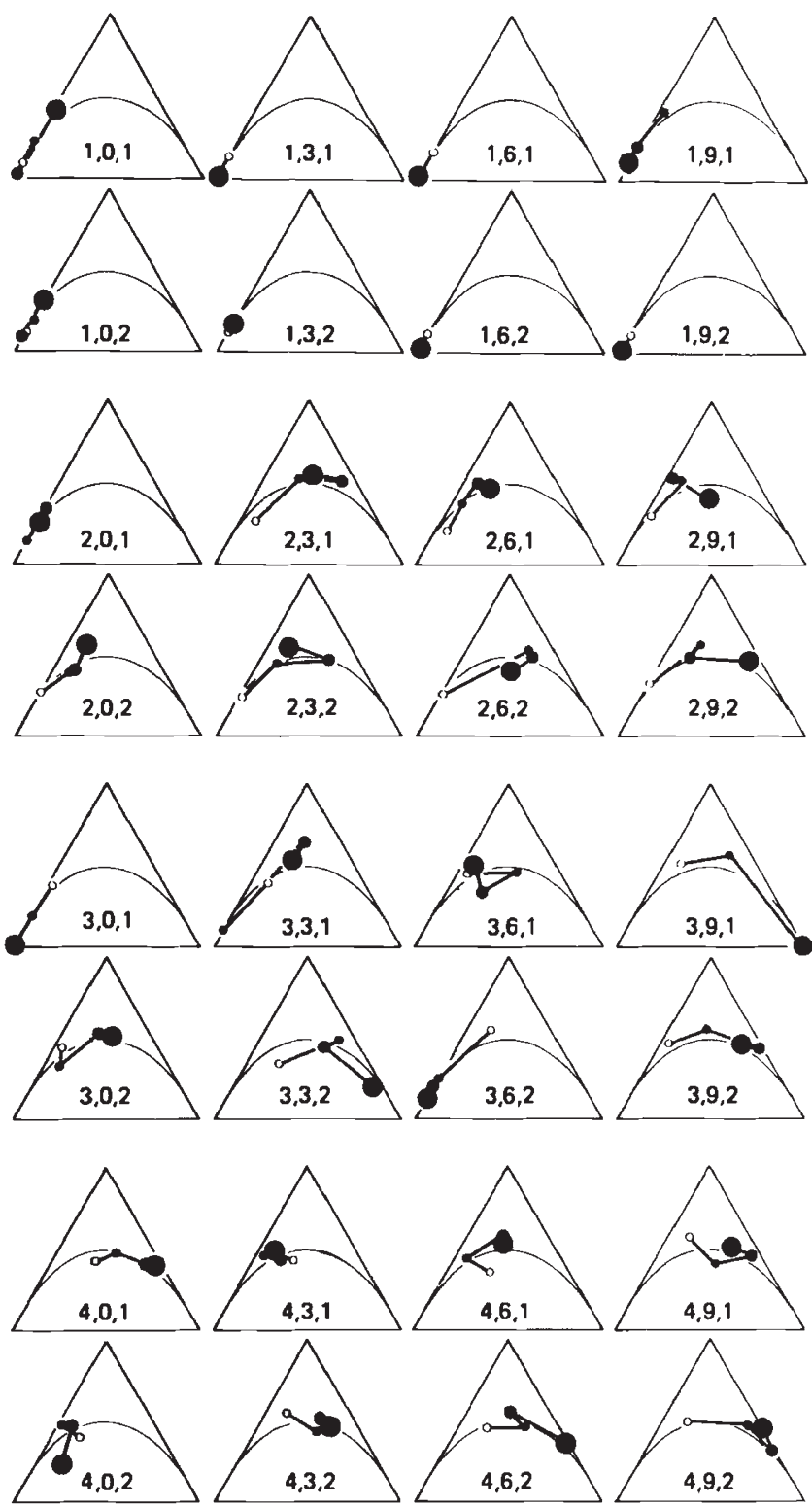

Fig. 2. De Finetti diagrams of the Adh genotype and allele frequency changes over the 30 generations in the 64 selection lines. Data for generation 0 are shown with small open circles and for generations 10,20 and 30 with successively larger closed circles. Genotype frequencies are represented in two dimensions with the left, right and top apices of each triangle corresponding to 100 per cent, $S S, F F$ and $F S$ respectively. Allele frequencies are represented unidimensionally and linearly, along the bottom of each triangle, with 100 per cent $S$ and $F$ on the left and right apices respectively. Parabolas representing Hardy-Weinberg genotype frequencies are also shown. 

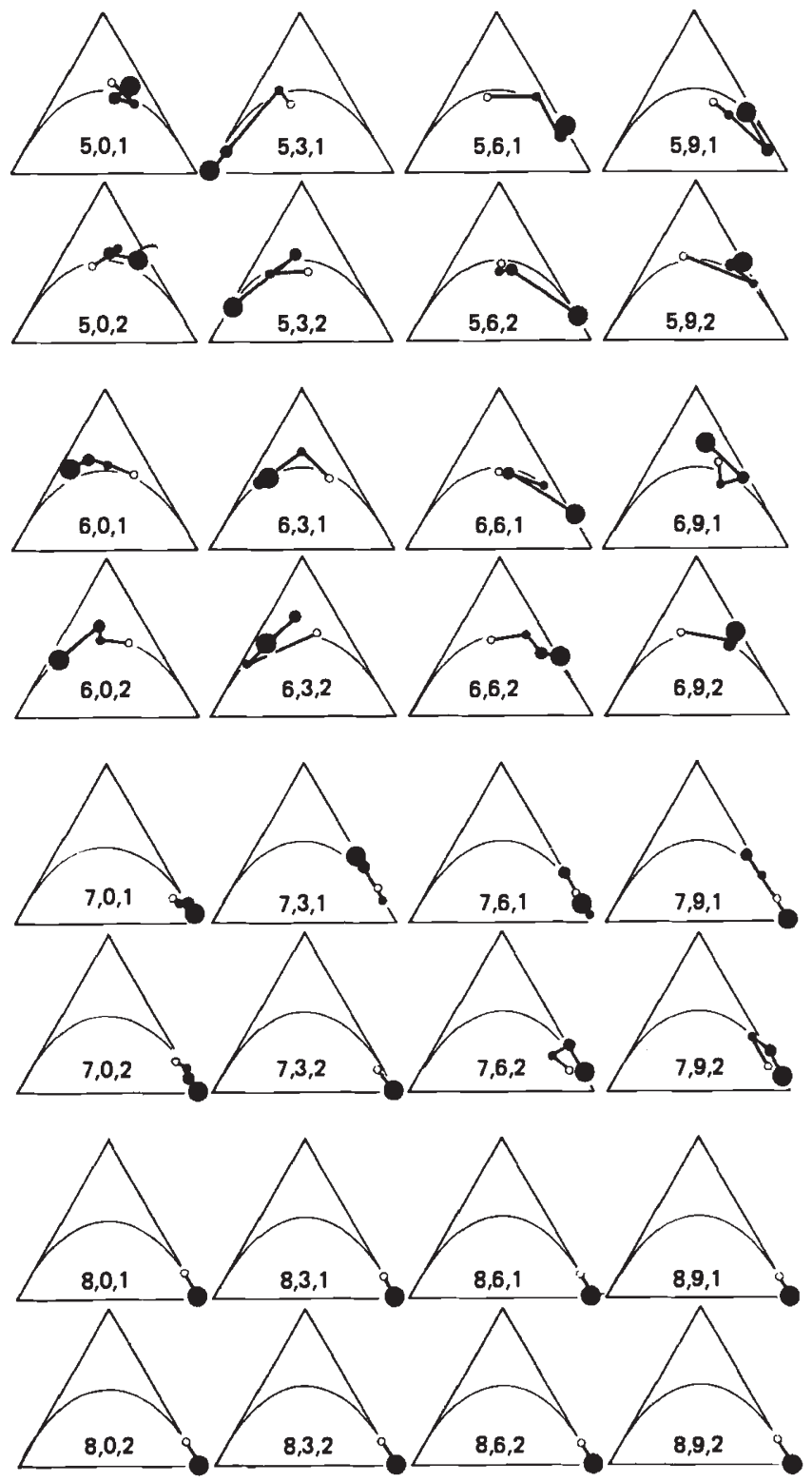

Fig. 2. cont.

excesses and the rises in $F$ frequency overall, it is obvious that any selection affecting $A d h$ must be relatively weak, or the effective population sizes relatively small, or both.

Table 3 gives the deviance decreases for a series of successively more parameterised models of selection on $A d h$ in the 64 selection lines. In the absence of significant effects due to the additional factor in the more parameterised model, each of these deviance decreases is expected to be 


\section{TABLE 3}

Deviance differences for testing the improvements in fit of a series of successively more parameterised models of selection to explain the observed Adh allele frequency changes. $f$ is the ratio of effective to observed population size assumed during model fitting. $(\cdot, \cdot, \cdot)=$ common, non $=$ zero $\mathrm{s}$ and $\mathrm{t}$ over all 64 lines; $(\mathrm{b}, \cdot, \cdot)=\mathrm{s}$ and $\mathrm{t}$ vary across the 8 base populations; $(\mathrm{b}, \mathrm{e}, \cdot)=\mathrm{s}$ and $t$ vary across the 4 ethanol environments within base populations; $(b, e, r)=s$ and $t$ vary across replicates within base population-environment combinations

\begin{tabular}{lrlcr} 
& & \multicolumn{3}{c}{ Deviance difference } \\
\cline { 3 - 5 } Model & df & $f=0 \cdot 1$ & $f=0 \cdot 5$ & $f=0 \cdot 9$ \\
\hline$(\cdot, \cdot, \cdot)$ & 2 & $22 \cdot 2^{* * *}$ & $28 \cdot 6^{* * *}$ & $42 \cdot 5^{* * * *}$ \\
$(b, \cdot, \cdot)$ & 14 & $85 \cdot 8^{* * *}$ & $158 \cdot 2^{* * *}$ & $242 \cdot 0^{* * *}$ \\
$(b, e, \cdot)$ & 48 & $62 \cdot 5 \dagger$ & $100 \cdot 1^{* * *}$ & $154 \cdot 6^{* * *}$ \\
$(b, e, r)$ & 64 & $24 \cdot 3$ & $45 \cdot 3$ & $71 \cdot 6$ \\
\hline
\end{tabular}

$\dagger P<0 \cdot 10,{ }^{* * *} P<0.001$.

approximately distributed as a $\chi^{2}$ (Wilson and Oakeshott, 1984). The deviance decreases also increase with the effective population size assumed; however, in the present data the significance or otherwise of deviance differences generally applied throughout the range of biologically reasonable effective population sizes (see below). Thus, a two parameter $(\cdot, \cdot, \cdot)$ selection model of common, non-zero $s$ and $t$ over all 64 selection lines gave a significantly better fit to the data than a neutral model with zero $s$ and $t$. A further significant improvement in fit was made by a 16 parameter $(b, \cdot, \cdot)$ selection model allowing $s$ and $t$ to vary among the eight base populations. And a still better fit was obtained with a 64 parameter $(b, e, \cdot)$ selection model in which $s$ and $t$ could vary across the four ethanol environments within each base population (although at $f=0.1, P \simeq 0.08$ ). However, a full, 128 parameter $(b, e, r)$ selection model in which $s$ and $t$ could vary between the two replicates in each of the 32 base population-ethanol environment combinations did not significantly improve the fit any further, even if the ratio, $f$, of effective to observed population size, was presumed to be as high as 0.9 .

Inspection of the contributions made by the eight base populations to the deviance difference between the $(b, \cdot, \cdot)$ and $(b, e, \cdot)$ models revealed that over half of the difference (e.g., $65 \cdot 1$ of the $100 \cdot 13$ at $f=0.5)$ was due to ethanol effects in a single base population, Brisbane $(b=3)$. Therefore

\section{TABLE 4}

Deviance differences for models of selection on the Adh allele frequency changes excluding the 16 Brisbane selection lines

\begin{tabular}{lrlcc} 
& & \multicolumn{3}{c}{ Deviance difference } \\
\cline { 3 - 5 } Model & df & $f=0 \cdot 1$ & $f=0.5$ & $f=0.9$ \\
\hline$(\cdot, \cdot, \cdot)$ & 2 & $14 \cdot 4^{* * *}$ & $24 \cdot 1^{* * *}$ & $36 \cdot 9^{* * * *}$ \\
$(b, \cdot, \cdot)$ & 12 & $80 \cdot 0^{* * *}$ & $140 \cdot 3^{* * *}$ & $214 \cdot 3^{* * *}$ \\
$(b, e, \cdot)$ & 42 & $13 \cdot 2$ & $32 \cdot 4$ & $44 \cdot 4$ \\
$(b, e, r)$ & 56 & 18.9 & $29 \cdot 5$ & $55 \cdot 8$ \\
\hline
\end{tabular}


the full analysis of deviance in table 3 was repeated excluding all Brisbane lines. The results (table 4 ) showed the same pattern of significant differences between models as was found among the total data, except that the $\left(b, e,{ }^{*}\right)$ model was no longer significantly better than $(b, \cdot, \cdot)$, even at $f=0.9$. With the exception of Brisbane, then, there were no significant selective effects of ethanol environments on $A d h$ allele frequencies.

Fig. 3 gives the confidence contours for the selection coefficients $s$ and $t$ in each of the $32 b-e$ combinations. The coefficients $s$ and $t$ define a set of relative fitnesses of $1: 1+t: 1+s$ for $S S: F S: F F$. All contours were calculated for $f=0.5$ and a subset were calculated for $f=0 \cdot 2$. These two $f$ values were used because Monte Carlo simulations carried out as in Wilson and Oakeshott (1984) suggested that the 95 per cent confidence interval for $f$ was from about 0.2 to about 0.5 . It is noteworthy that Wilson and Oakeshott (1984) determined the same interval in their experiment.

There was relatively little difference between the contours calculated at $f=0.2$ and 0.5 , although most of the contours defined rather broad ranges of $s$ and $t$ values. (This was particularly true for Palm Woods, Melbourne and St Peters ( $b=1,7$ and 8$)$, where generation $0 F$ frequencies had been most extreme.) The lack of definition reflected the facts that each contour was based on only two replicates and that the 95 per cent confidence interval for effective population size was only about 15 to 40 flies (based on the interval for $f$ above).

Consistent with the analyses of deviance in tables 3 and 4, the only base population for which there were clear differences between the contours for different ethanol environments was Brisbane, where the value of $s$ was significantly greater than 0 for 9 per cent selection lines, but not for 0 per cent, 3 per cent or 6 per cent lines. This indicated that the $F F$ homozygote was significantly fitter than $S S$ on 9 per cent ethanol, but not at lower ethanol concentrations.

Fig. 4 gives the confidence contours for $s$ and $t$ for each of the eight base populations calculated over all ethanol environments. Each of these contours was based on eight selection lines and, with the exceptions of Palm Woods, Melbourne and St Peters, $s$ and $t$ were relatively tightly defined. The contours revealed two general points about $s$ and $t$. First, $t$, for the fitness of $F S$ relative to $S S$, was significantly positive for all base populations except Palm Woods, Melbourne and St Peters. This trend for positive $t$ was also true in the $s$ and $t$ contour for the total data over all 64 lines (fig. 4), where the maximum likelihood estimate was $s=+0 \cdot 07, t=+0 \cdot 12$.

The second general point from fig. 4 was that $s$, for the fitness of $F F$ relative to $S S$, was greater for base populations with higher generation 0 $F$ frequencies. Indeed the same trend was obvious if the equilibrium $F$ frequencies predicted by the maximum likelihood $s$ and $t$ estimates were plotted against generation $0 F$ frequencies (fig. 5). In general, the selection operating over the 30 generations in the experimental populations worked to retain $F$ frequencies around (generally slightly above) the values originally found in the source populations in the wild.

\section{Discussion}

Before considering the responses of the Adh polymorphism, it is important to note that after the thirty generations differences in ethanol tolerance 


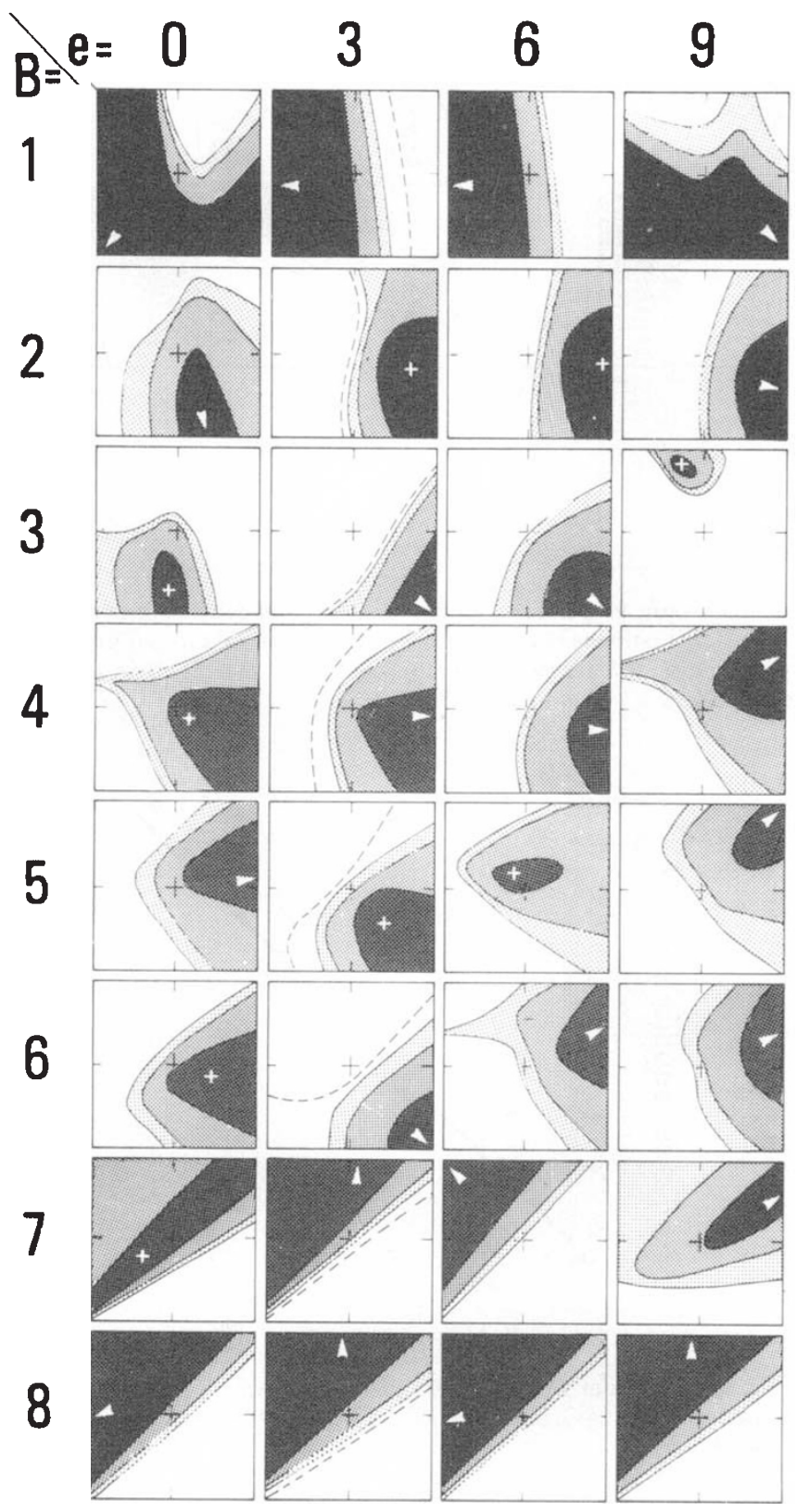

Fig. 3. The 50 per cent, 95 per cent and 99 per cent confidence contours for the maximum likelihood $s$ and $t$ estimates in each of the 32 base population-environment combinations. Values of $s$, for the fitness of $F F$ relative to $S S$, are given along the $\mathrm{Y}$ axis. Values of $t$, for the fitness of $F S$ relative to $S S$, are given along the $X$ axis. The scale for $t$ (or $s$ ) is from -0.5 on the left (or bottom) to +0.5 on the right (or top) of each graph. The best estimate for each population is given by a cross (or an arrow if off-scale) and the three confidence intervals are represented with successively lighter shades of stipple. All contours are calculated for $f=0.5$ except that illustrative $50 \%$ contours for $f=0.2$ are also shown (broken lines) for the eight $e=3 \%$ graphs. 

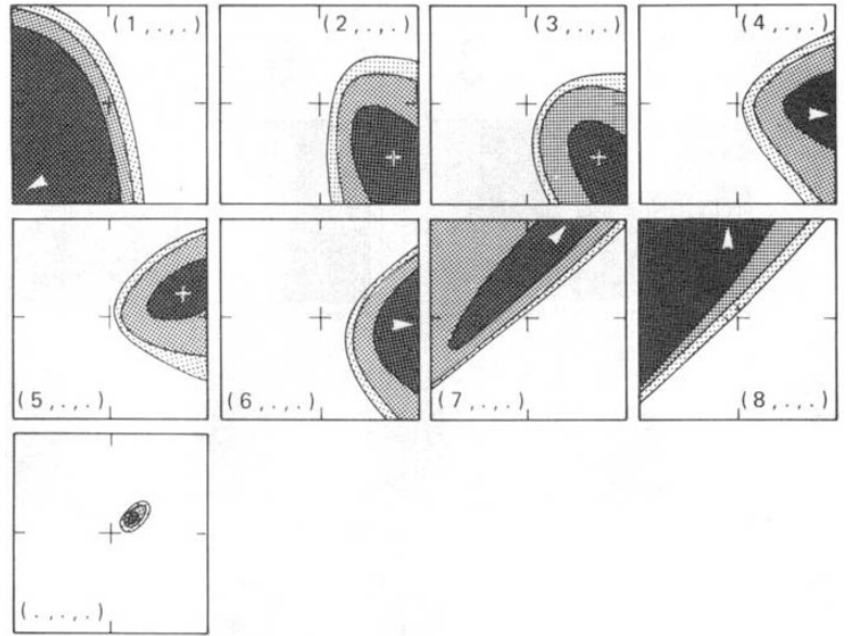

FIG. 4. The 50 per cent, 95 per cent and 99 per cent confidence contours for the maximum likelihood $s$ and $t$ estimates in each of the eight base populations (numbered in increasing order of generation $0 F$ frequency) and in the total data over all 64 selection lines .,., . Format as in fig. 3.

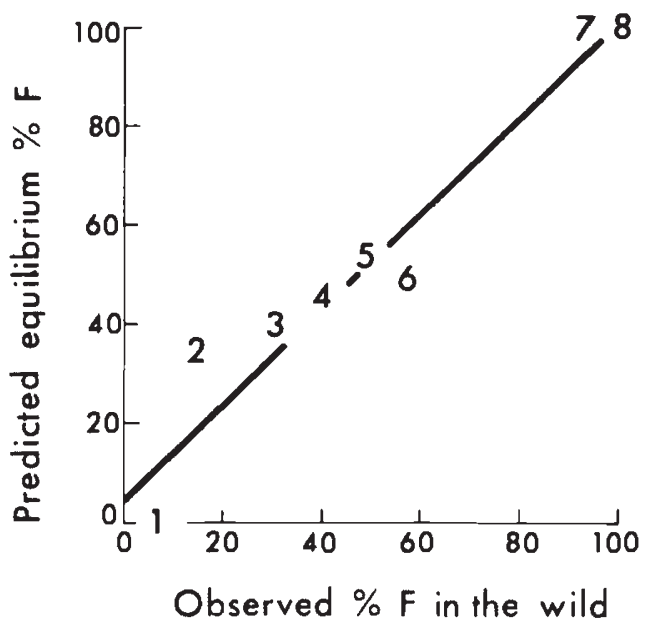

FIG. 5. Graph of equilibrium $F$ frequencies predicted by the $s$ and $t$ estimates in fig. 4 for the eight base populations against the $F$ frequencies observed in their source populations from the wild.

had developed between selection lines maintained in the four ethanol environments. Moreover these differences were consistent across the eight base populations used. Thus, the experiment had successfully established differences in ethanol tolerance among groups of selection lines in which the $A d h$ polymorphism might a priori be expected to be involved.

Indeed the potential for differences in $A d h$ responses was enhanced by the opposite tolerance responses of the 3 per cent versus 6 per cent and 9 per cent selection lines. The survival rates of adults and preadults after test 
doses of 9 per cent ethanol were higher in the 6 per cent and 9 per cent selection lines than in the 0 per cent lines. On the other hand, the 3 per cent lines were similar to the 0 per cent lines in adult female survival and lower in egg-to-adult and adult male survival after the test dose. Analogous discrepancies between the responses of the 3 per cent versus 6 per cent and 9 per cent lines will be reported elsewhere for developmental times, productivities and adult dry weights after the test doses. This is consistent with other evidence from fitness component analyses at different ethanol concentrations that 3 per cent ethanol is a metabolic benefit to $D$. melanogaster, whereas 6 per cent and 9 per cent are metabolic costs (Oakeshott et al., 1980; Parsons and Stanley, 1981).

Despite the extent of the tolerance differences which developed among lines selected on the four ethanol environments, and the consistency of these differences across the eight base populations, the only response of the $A d h$ locus was an increased fitness of $F F$ relative to $S S$ in the 9 per cent versus 0,3 and 6 per cent Brisbane lines. This result for Brisbane is the only one consistent with the increased $F$ frequency previously reported in many populations kept on ethanol food. Yet, of the eight base populations we used, Brisbane was not distinguished by extremes of $\ln (2 L) t$ or FCh.D. frequencies, ADH activities or obvious features of the collection locality (table 1). The general result from most of our lines is clearly that $F$ frequency does not increase in response to selection on ethanol media.

This result is in direct contrast to those reported in some previous studies and in an attempt to resolve this discrepancy we have collated details of all the published multi-generation experiments investigating $A d h$ allele frequencies on ethanol supplemented food (table 5). Of the 30 base populations studied, nine showed elevated $F$ frequencies on ethanol food, 21 showed no significant difference from controls and none showed a significant decrease in $F$ frequency. The outcome was not obviously related to the ethanol concentration, base population size or initial $F$ frequency. However, it was strongly correlated with the origin of the base populations and with the time between their capture and the start of the experiment. With few exceptions the only base populations showing elevated $F$ frequencies on ethanol media were non-Australasian collections which had spent three or more years in the laboratory prior to the experiment.

It is unclear simply from this whether the continental origin of the populations or their capture-to-test time more closely reflects the causal factors. However other lines of evidence suggest that the continental origin is not relevant. Thus, if $A d h$ genotypes are extracted from highly inbred or long established laboratory populations, then characteristic differences in in vitro $\mathrm{ADH}$ activity and ethanol tolerance can be found between them which are similar for Australasian, American and European populations (van Delden, 1982 for references). Moreover, $F$ and $S$ variants extracted from Australasian populations have identical amino acid sequences to their American and European counterparts (Kreitman, 1983). We therefore suspect that the failure of the $A d h$ polymorphism to respond to ethanol selection in most base populations noted in table 5 is causally related not to their Australasian origin but to their relatively short capture-to-test time (within 4 months).

A role of capture-to-test time might reflect changes in background genotype after wild/caught populations are brought into the laboratory. 


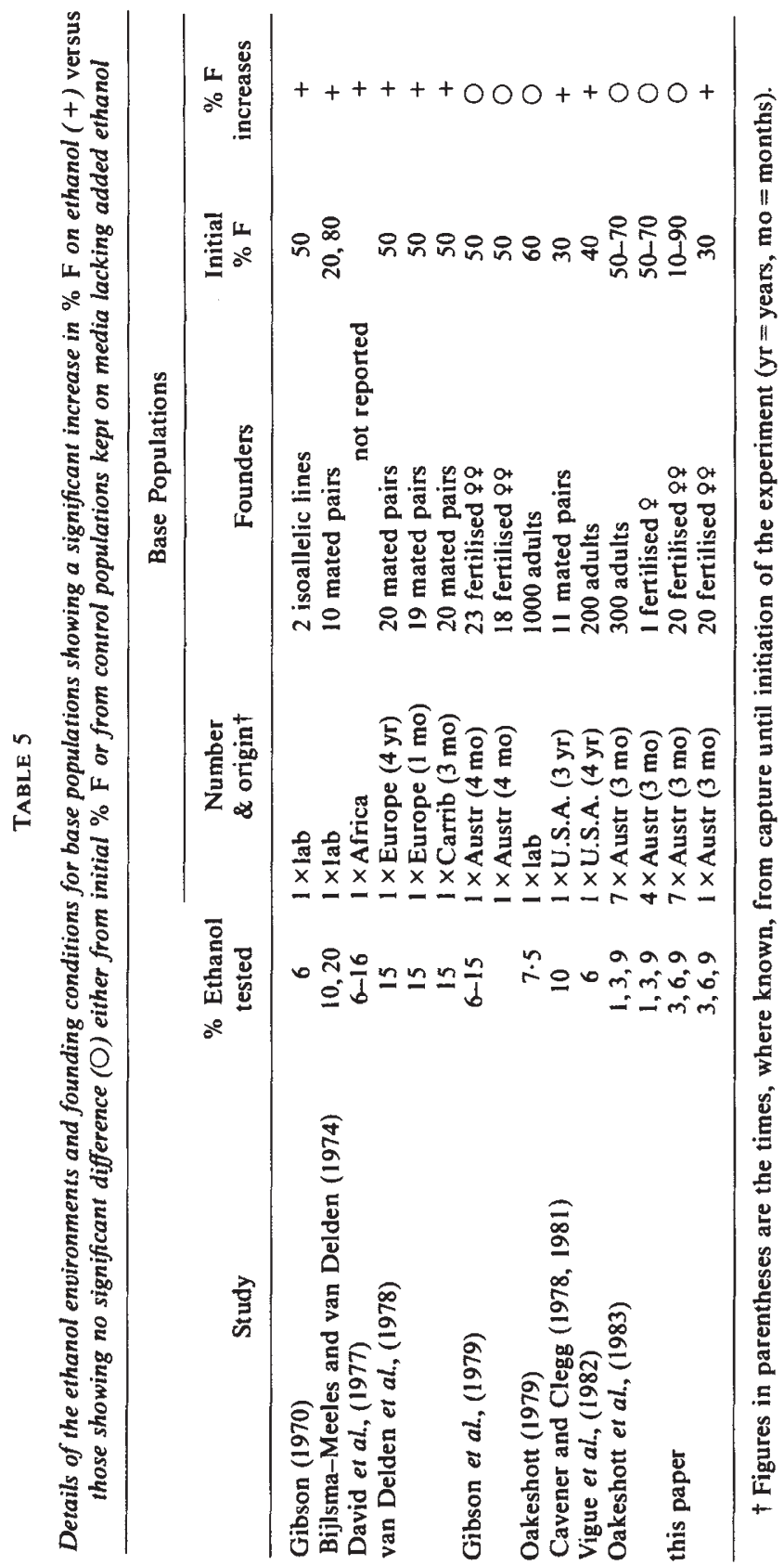


Whereas rotting fruits can contain up to 3 per cent ethanol, laboratory food is virtually free of ethanol, unless specifically supplemented (Gibson et al., 1981; Oakeshott et al., 1982b). Adh frequencies in long established laboratory populations might respond to selection on ethanol media because the genetic architecture evolved in natural populations for adaptation to ethanol has been changed.

Whatever the reasons for the discrepancies among studies it is now clear that in the majority of base populations most closely related to their wild source populations the $A d h$ polymorphism does not respond to selection on ethanol media. This is consistent with the recent finding by Middleton and Kacser (1983) that in vivo degradation rates of ingested ethanol do not differ among the $F F, F S$ and $S S$ genotypes. In his review of the population genetics of $A d h$, van Delden (1982) concluded

"It appears that the $A d h$ polymorphism meets the demands made for the direct detection of selection in view of the relation between the enzyme activities in vitro of the genotypes and their survival in the presence of ethanol."

Van Delden (1982) admitted that several studies (reviewed in our Introduction) finding no relation between geographic variation in $A d h$ allele frequencies and ethanol tolerance in wild communities were difficult to interpret in this light. It now seems that selection among the $A d h F$ and $S$ alleles in response to ethanol is largely restricted to laboratory-adapted populations, the relevance of which to wild populations has not been demonstrated.

Although the selection on Adh generally did not differ among the four ethanol environments tested here, it differed very significantly among the eight base populations. In each base population selection operated to stabilise $F$ frequency in the vicinity of, or slightly above, that observed in the original collection. Similar results were obtained by Berger (1971) and Wilson et al. (1982), who found that, in populations recently collected from the wild, experimentally perturbed $F$ and $S$ frequencies returned over a number of generations to values around those in the original collections. These results indicate that components of the genetic background influence any selection on $A d h$, not only specifically in relation to ingested ethanol, but also, more broadly, irrespective of added ethanol. In the present study any direct selection on $A d h$ due to ethanol was generally insufficient to overcome the stabilising selective forces imposed by the genetic background.

Despite the effects of the genetic background, one aspect of the selection affecting Adh which was consistent across all the base populations and ethanol environments studied here was heterozygote advantage. This was evident not only in the highly significant excesses of FS above HardyWeinberg expectations, but also in the consistently greater estimates of fitness for FS over both homozygotes. It was also apparent in the fitness set of $1 \cdot 00: 1 \cdot 12: 1 \cdot 07$ for $S S: F S: F F$ which was estimated from the total data. This general superiority of heterozygotes concurs with the FS excesses observed by van Delden et al., (1978) in their study of laboratory populations and by Franklin (1981) in his five year survey of seven Australian winery populations. However, the in vivo and in vitro functions of ADH in which such heterozygote advantage might accrue have yet to be identified. 
Acknowledgements. We thank Ms R. G. Attewell, Ms A. V. Wilks and Mr D. A. Willcocks for excellent technical assistance and Drs P. R. Anderson, I. A. Boussy, F. M. Cohan, S. Easteal and W. R. Knibb for valulable discussions and comments on the manuscript.

\section{REFERENCES}

ANDERSON, D. G. 1982. Alcohol dehydrogenase activity and ethanol tolerance along the ADH cline in Australia. In Lakovaraa, S. (ed) Advances in Genetics, Development, and Evolution of Drosophila, Plenum, New York, pp. 263-272.

BERGER, E. M. 1971. A temporal survey of allelic variation in natural and laboratory populations of Drosophila melanogaster. Genetics, 67, 121-136.

BIJLSMA-MEELES, E. AND VAN DELDEN, w. 1974. Intra and inter-population selection concerning the alcohol dehydrogenase locus in Drosophila melanogaster. Nature, 247, 369-371.

BRISCOE, D. A., ROBERTSON, A. AND MALPICA, J. 1975. Dominance at Adh locus in response of adult Drosophila melanogaster to environmental alcohol. Nature, 255, 148-149.

CAVENER, D. G. AND CLEGG, M. T. 1978. Dynamics of correlated genetic systems. IV. Multilocus effects of ethanol stress environments. Genetics, 90, 629-644.

CAVENER, D. G. AND CLEGG, M. T. 1981. Multigenic response to ethanol in Drosophila melanogaster. Evolution, 35, 1-10.

CHAMBERS, G. K., LAVER, W. G., CAMPBELL, S. AND GiBSON, J. B. 1981. Structural analysis of an electrophoretically cryptic alcohol dehydrogenase variant from an Australian population of Drosophila melanogaster. Proc. Natl. Acad. Sci. USA, 78, 3103-3107.

DAVID, J. R. 1982. Latitudinal variability of Drosophila melanogaster: allozyme frequencies divergence between european and afrotropical populations. Biochem. Genet., 20, 747-761.

DAVID, J. R. AND BOCQUET, C. 1975. Similarities and differences in latitudinal adaptation of two Drosophila sibling species. Nature, 257, 588-590.

DAVID, J., BOCQUET, C., FOUILLET, P. AND ARENS, M-F. 1977. Tolérance gééétique à l'alcohol chez Drosophila: comparaison des effets de la sélection chez $D$. melanogaster et $D$. simulans. C. R. Acad. Sci. Paris Serie D, 285, 405-408.

FRANKLIN, I. R. 1981. An analysis of temporal variation at isozyme loci in Drosophila melanogaster. In Gibson, J. B. and Oakeshott, J. G. (eds) Genetic Studies of Drosophila Populations, Australian National University, Canberra, pp. 217-236.

GIBSON, J. 1970. Enzyme flexibility in Drosophila melanogaster. Nature, 227, 959-961.

GIBSON, J. B., LEWIS, N., ADENA, M. A. AND ẆILSON, S. R. 1979. Selection for ethanol tolerance in two populations of Drosophila melanogaster segregating alcohol dehydrogenase allozymes. Aust. J. Biol. Sci., 32, 387-398.

GIBSON, J. B., MAY, T. W. AND WILKS, A. V. 1981. Genetic variation at the alcohol dehydrogenase locus in Drosophila melanogaster in relation to environmental variation: ethanol levels in breeding sites and allozyme frequencies. Oecologia, 5l, 191-198.

GIBSON, J. B. AND OAKESHOTT, J. G. 1982. Tests of the adaptive significance of the alcohol dehydrogenase polymorphism in Drosophila melanogaster: paths, pitfalls and prospects. In Barker, J. S. F. and Starmer, W. T. (eds.) Ecological Genetics and Evolution, Academic Press, New York, pp. 291-306.

HICKEY, D. A. AND MCLEAN, M. D. 1980. Selection for ethanol tolerance and Adh allozymes in natural populations of Drosophila melanogaster. Genet. Res., 36, 11-15.

JOHNSON, F. M. AND SCHAFFER, H. E. 1973. Isozyme variability in species of the genus Drosophila. VII. Genotype-environment relationships in populations of $D$. melanogaster from the Eastern United States. Biochem. Genet., 10, 149-163.

KNIBB, W. R. 1983. Chromosome inversion polymorphisms in Drosophila melanogaster. III. Gametic disequilibria and the contributions of inversion clines to the ADH and GPDH allozyme clines in Australasia. Genetica, 61 139-146.

KNIBB, W. R., OAKESHOTT, J. G. AND GIBSON, J. B. 1981. Chromosome inversion polymorphisms in Drosophila melanogaster. I. Latitudinal clines and associations between inversions in Australasian populations. Genetics, 98, 833-847.

KREITMAN, M. 1983. Nucleotide polymorphism at the alcohol dehydrogenase locus of Drosophila melanogaster. Nature, 304, 412-417.

LEWIS, N. AND GIBSON, J. B. 1978. Variation in amount of enzyme protein in natural populations. Biochem. Genet., 16, 150-169.

MALPICA, J. M. AND VASSALLO, J. M. 1980. A test of the selective origin of environmentally correlated allozyme patterns. Nature, 286, 407-408. 
MARKS, W., BRITTNACHER, J. G., MCDONALD, J. F., PROUT, T. AND AYALA, F. J. 1980. Wineries, Drosophila, alcohol and Adh. Oecologia, 47, 141-144.

McKenZIE, J. A. AND McKECHNIE, S. W. 1981. Ethanol tolerance and the Adh polymorphism in a natural population of Drosophila melanogaster. Nature, 272, 75-76.

MIDDLETON, R. J. AND KACSER, H. 1983. Enzyme variation, metabolic flux and fitness: alcohol dehydrogenase in Drosophila melanogaster. Genetics, 105, 633-650.

OAKESHOTT, J. G. 1979. Selection affecting enzyme polymorphisms in laboratory populations of Drosophila melanogaster. Oecologia, 43, 341-354.

OAKESHOTT, J. G. AND GIBSON, J. B. 1981. Is there selection by environmental ethanol on the alcohol dehydrogenase locus in Drosophila melanogaster? In Gibson, J. B. and Oakeshott, J. G. (eds.) Genetic Studies of Drosophila Populations, Australian National University, Canberra, pp. 103-120.

OAKESHOTT, J. G., GIBSON, J. B., ANDERSON, P. R. AND CHAMP, A. 1980. Opposing modes of selection on the alcohol dehydrogenase locus in Drosophila melanogaster. Aust. J. Biol. Sci., 33, 105-114.

OAKESHOTT, J. G., GIBSON, J. B., ANDERSON, P. R., KNIBB, W. R., ANDERSON, D. G. AND CHAMBERS, G. K. 1982a. Alcohol dehydrogenase and glycerol-3-phosphate dehydrogenase clines in Drosophila melanogaster on different continents. Evolution, 36, 86-96.

OAKESHOTT, J. G., MAY, T. W., GIBSON, J. B. AND WILLCOCKS, D. A. 1982 b. Resource partitioning in five domestic Drosophila species and its relationship to ethanol metabolism. Aust. J. Zool., 30, 547-556.

OAKESHOTT, J. G., WILSON, S. R. AND GIBSON, J. B. 1983. An attempt to measure selection coefficients affecting the alcohol dehydrogenase polymorphism in Drosophila melanogaster populations maintained on ethanol media. Genetica, 61, 151-159.

PARSONS, P. A. AND STANLEY, S. M. 1981. Comparative effects of environmental ethanol on Drosophila melanogaster and $D$. Simulans adults, including geographic differences in $D$. melanogaster. In Gibson, J. B. and Oakeshott, J. G. (eds.) Genetic Studies of Drosophila Populations, Australian National University, Canberra, pp. 47-57.

VAN DELDEN, w. 1982. The alcohol dehydrogenase polymorphism in Drosophila melanogaster. Evol. Biol., 15, 187-222.

VAN DELDEN, W., BOEREMA, A. C. AND KAMPING, A. 1978. The alcohol dehydrogenase polymorphism in populations of Drosophila melanogaster. I. Selection in different environments. Genetics, 90, 161-191.

VIGUE, C. L., WEISGRAM, P. A. AND ROSENTHAL, E. 1982. Selection at the alcohol dehydrogenase locus of Drosophila melanogaster: effects of ethanol and temperature. Biochem. Genet., 20, 681-688.

WILKS, A. V., GIBSON, J. B., OAKESHOTT, J. G. AND CHAMBERS, G. K. 1980. An electrophoretically cryptic alcohol dehydrogenase variant in Drosophila melanogaster. II. Post electrophoresis heat-treatment screening of natural populations. Aust. J. Biol. Sci., 34, 625-637.

WILSON, S. R., OAKESHOTT, J. G., GIBSON, J. B. AND ANDERSON, P. R. 1982. Measuring selection coefficients affecting the alcohol dehydrogenase polymorphism in Drosophila melanogaster. Genetics, 100, 113-126.

WILSON, S. R. AND OAKESHOTT, J. G. 1984. Statistical analysis of data from gene frequency perturbation experiments. Biometrical Journal, (in press). 